\title{
Metabolic competition the tumor - tumor-bearing: Using the amino acids at doses comparable to their physiological levels
}

\author{
Nefyodov $L^{1 *}$, Karavay $A^{2}$, Karavay $\mathbf{P}^{2}$, Karavay $\mathbf{N}^{3}$ and Koliada $\mathbf{T}^{3}$ \\ ${ }^{1}$ Department of Biochemistry of Yanka Kupala Grodno State University, Belarus \\ ${ }^{2}$ Department of Oncology of Grodno State Medical University, Belarus \\ ${ }^{3}$ Department of Hyperbaric Oxygenation of Grodno Regional Clinical Hospital, Belarus
}

\begin{abstract}
On the basis of the experimental data we suggest that the differences discovered in certain amino acids concentrations in blood plasma, red blood cells and tumors are criteria in early diagnostics of primary cancerous growth as well as in estimation of the efficacy of specific cancer treatment. Clinical studies on biological fluids and tumors of 1,495 patients with cancer of the mammary gland, lungs, prostate, ovaries, bladder or digestive tract showed significant changes in physiological concentrations of amino acids which either directly or indirectly regulate processes of antitumor response, oncogenesis, immunogenesis and apoptosis were shown. Our strategy of application of amino acids as medicinal preparations includes a targeted effect on the functional and metabolic relationships which are changed in specific pathology through the effect on the regulatory mechanisms of intermediate metabolic reactions, limiting stages of metabolic flows, utilization of energy substrates and transport systems restricting the processes of amino acids pool formation. The creation methodology of pathogenetic compositions of amino acids and their derivatives on the basis of their physiological concentration for practical application of their regulatory effects in oncology was discussed.
\end{abstract}

\section{Introduction}

Amino acids and their derivatives are mostly universal natural regulators and endogenous modifiers of biological reactions. However, numerous biological properties of these compounds as drugs were used for correction of deficiencies or realization of pharmacological and immediate metabolic effects, disregarding a regulatory action. Amino acid profile indices that allow early detection of diseases, which would provide time for intervention before irreversible damage occurred, are being created. Thus, amino acid profiles represent biomarkers for diseases or deviations from a normal state of health. Our array technology will play an important role in metabolomics in biomarker discovery, clinical medicine, including cancer as well as, at other stages of drug discovery and development (for example, target discovery, mechanism of action or predicting toxicity) [1-6].

Changes in amino acid pool in liquids and tissues of patients specifically characterize development of cancer. Correction of the intermediate metabolic changes in cancer can be reached by the use of certain amino acids or their combinations [7-9].

At present, there are the following main lines in exploitation of biochemical (metabolic) properties of amino acids and their derivatives in clinical practice:

- Use of amino acids or multicomponent mixtures of amino acids (mainly, essential elements combined with vitamins and trace elements) for replacement therapy or deficiency of essential nutrients and proteins.

- Use of drugs containing individual amino acids or their compositions, designed on the basis of their additive functional and metabolic action, which "exploits" pharmacological activity (effects of activation of redox processes, reactions of energy metabolism and neutralization of xenobiotics compounds) of this class [10-12].

However, the use of certain levels of L-amino acids or their compositions or their deficiency implements direct pharmacological effects that practically ignore their regulatory effects on metabolic processes and key metabolic reactions.

To understand the metabolic processes and vital functions of the regulatory effect of amino acids which manifests itself under natural or near concentrations of these compounds in body fluids and tissues. It is obvious that the effective use of L-amino acids or their derivatives for metabolic correction and directional changes in metabolism under pathological or extreme conditions is limited by insufficient accumulation of information about the key mechanisms of regulatory effects of the compounds tested at concentrations comparable to their physiological (endogenous) levels [1,2,6,9-12].

\section{Materials and methods}

Our development methodology is based on:

- Studies on physiological concentrations of free amino acids, their derivatives, precursors and metabolites, as well as biochemical marker

Correspondence to: Nefyodov L, Department of Biochemistry, Yanka Kupala Grodno State University, Belarus, E-mail: 1.nefyodov@mail.ru

Key words: free amino acids, regulatory effects, oncology

Received: April 19, 2017; Accepted: May 16, 2017; Published: May 19, 2017 
parameters in healthy donors and patients with various pathologies.

- Creation of a unified database for the parameters investigated, construction of an empirical mathematical model consisting of pathogenic markers of specific pathology and amino acid profiles.

- Specialized development of new formulations of the compositions of infusion solutions of amino acids and their derivatives [1,2,5,9-12].

The results and our concept are based on research into formation of free amino acid pool in biological liquids and tissues of 1,495 of patients with cancer of the mammary gland, lungs, prostate, ovaries, bladder or digestive tract.

\section{Results and discussion}

Numerous results of determination of amino acids and their derivatives in human body fluids and tissues [3] allowed systematization of the accumulated data and identified the areas for exploitation of their metabolic effects, primarily in laboratory diagnostics, and application in clinical practice as drugs [4].

In view of the fact that free amino acids are represented by a wide range of related chemical structures and metabolic transformations of compounds that are formed in the body fluids and tissues, the findings obtained proved that quantification of amino acid pool contributes to the diagnosis of various diseases, including hepatobiliary pathology, cardiovascular and immune systems, oncological cases, cerebrovascular pathology, as well as alcoholism and diabetes [1,2,4,9-11]. It turned out that the vast majority of the diagnostic values of the group had alterations in the levels of functionally and metabolically related amino acids and their derivatives while no specific changes, as such, were observed in the concentrations of individual compounds of this class.

Metabolically related amino acids and their derivatives had the nature of amino acid profiles of body fluids and tissues of animals and humans when compared with the use of a multivariate analysis and mathematical modeling. At the same time, it was convincingly demonstrated that removal or correction of intermediate metabolic changes can be achieved using individual amino acids and their derivatives, or a combination of them as universal natural bioregulators - compounds that at physiological concentrations have a direct effect on the mechanisms of cellular metabolism $[5,6,12]$.

By now, there is some evidences for the importance of not only amino acids as building blocks for protein synthesis, but also regulators of gene expression at the level of mRNA translation by mTORdependent mechanism, signaling molecules and biological response modifiers, as well as precursors of a wide range of bioregulators, which play a key role in the integration of major metabolic fluxes [13-18].

Based on the positions of metabolomics, the free amino acid pool in biological fluids and tissues is regarded as a single information unit which is a kind of "a chemical projection" of the genome, the proteome realized through this approach not only develops ideas about the pool of amino acids as a dynamical system-generated supply of them from outside, but also due to endogenous synthesis, transport, degradation and excretion and allows the identification of "key points" in intermediate metabolic equilibrium shift that may reflect ratios at the individual levels of endogenous amino acids and related species (metabolically- related) compounds $[5,6,19,20]$.

We were the first to demonstrate that endogenous levels of free amino acids in fluids and tissues are the most important integral indicators and regulators of metabolism.
This enables us to prove the use of individual amino acids or their combinations for guided correction of metabolism under specific human diseases and significantly expands the area of practical application of these compounds as blood substitutes. The regulatory effects of amino acids contribute to understanding of their influence on biochemical processes and vital functions which is manifested at natural (endogenous) or close to natural concentrations of these compounds. A regulatory effect observed after administration of amino acids can be achieved by using either individual amino acids, or a combination of their small sets. The structure of these compositions in specific ratios may include almost any amino acids, their structural analogs or derivatives with a known mechanism of action.

The trend developed is notable since it allows to assess the pool of amino acids in biological fluids and tissues a single information unit and while analyzing it, it makes possible to estimate the segments which disturb the metabolic balance (changes in the metabolic flows ratio and their balance shifts).

Our methodology proposed for development of new formulations of multicomponent infusion solutions based on amino acids and related compounds for the correction of metabolic imbalance occurring in various diseases relies on the application of research results on the regularities of formation of amino acid pool in biological fluids and tissues under various pathological conditions. The composition and amount of highly purified amino acids in these infusion solutions should be determined primarily by their physiological (regulatory) concentrations, which distinguishes them from traditionally used amino acid solutions for parenteral nutrition, where the content of their components is calculated from daily requirements of the human body for them without due consideration for regulatory actions of the compounds administered.

In oncological practice, individual amino acids or their compositions should be applied according to their physiological concentrations and changes in the structure of the amino acid pool in patients. Amino acids with anti-carcinogenic effects may be leucine, tryptophane and taurine.

\section{Conclusion}

The methodology of development of new constituents of multicomponent infusion solutions offered by us on the basis of amino acids and their related connections is intended for correction of the metabolic imbalance arising in various diseases and is based on application of the results on research regularities of formation of amino acid pool in biological liquids and tissues of individuals under most various pathological states.

In oncological practice, certain amino acids or their compositions should be applied according to their physiological concentrations and changes in the structure of amino acids pool in patients. As a result of numerous experimental and clinical studies, we proved that diagnostically significant in oncology and "anticarcinogenic" are such amino acids as leucine, tryptophan and taurine.

The new methodology of development of minicomponent amino acid infusion solutions offered by us intended for correction of the metabolic imbalance arising in case of various localization and stages of malignant growth is based on the results of research into regularities of formation of amino-acid pool in biological liquids and tissues.

The ratios of the individual components and their amount in such mixtures should comply with their physiological (endogenous) concentrations in normal blood plasma and tissues. 


\section{References}

1. Nefyodov L (1999) Taurine (biochemistry, pharmacology, medical application) Grodno RIPH.

2. Nefyodov L (1996) Amino Acids And Their Derivatives (chemistry, biochemistry, pharmacology, medicine). Proc of Internat. Symp, Grodno.

3. Nefyodov LI, Karavay PA, Karavay NL (2014) Regulatory action of free amino acids and development on the basis of highly of substances infusion solutions with pathogenetic deterministic composition. Laboratory diagnosis Eastern Europe.

4. Holden JT (1962) Amino Acid Pools. Elsevier, Amsterdam

5. Lubec C (1990) Amino Acids (Chemistry, Biology, Medicine). Escom New York.

6. Nefyodov LI (2010) The results of biochemical research and development of nitrogencontaining compounds of natural origin: methodology of exploitation of biological properties as universal natural regulators of metabolism and drugs.

7. Nefyodov LI, Uglyanica KN, Smirnov VY, Doroshenko YM, Fomin KA, et al. (1996) Amino acids and their derivatives in tumour tissue from patients with breast cancer treated with Ukrain. Part VI. Drugs Exp Clin Res 22: 155-157.

8. Nefyodov L, Uglyanica KN, Smirnov VY, Doroshenko YM, Fomin KA, et al. (1996) Amino acids and their derivatives in blood plasma of patients with breast cancer treated with Ukrain. Part V. Drugs Exp Clin Res 22: 159-161.

9. Nefyodov LI (1999) Biological activity and transport of drugs Proc of Internat. Symp.; Grodno. $-1999 .-189 \mathrm{p}$.

10. Nefyodov L (2000) VI Ordinary General Assembly Society of Biochemistry of Belarus. Proc of Internat. Symp. Grodno.
11. Nefyodov L (2001) Amino acids and their derivatives in biology and medicine. Proc of Internat. Symp. - Grodno.

12. Nefyodov L (2001) Target - oriented regulation of metabolic equilibrium by amino acids and strategy of their application as drugs with directional effects. XXXVII Zjazd Polskiego towarzystwa biochemicznego, Torun.

13. Ihata Y, Miyagi E, Numazaki R (2013) Amino acid profile index for early detection of endometrial cancer: verification as a novel diagnostic marker. Int J Clin Oncol 19 : 364-372.

14. Fafournoux, P, Bruhat A, Jousse C (2000) Amino acid regulation of gene expression. BioChem J 351: 1-12.

15. Meijer A (2003) Amino acids as regulators and components of nonproteinogenic pathways. J Nutr 6: 2057S-2062S.

16. Bruhat A, Chérasse Y, Chaveroux C, Maurin AC, Jousse C, et al. (2009) Amino acids as reg- ulators of gene expression in mammals: molecular mechanisms. Biofactors 35 : 249-257.

17. Ananieva E (2015) Targeting amino acid metabolism in cancer growth and anti-tumor immune response. World J Biol Chem 6: 281-289.

18. Hong Ma (2014) Plasma free amino acid profiling of esophageal cancer using highperformance liquid chromatography spectroscopy. World J Gastroenterol 20: 8653 8659 .

19. Karavay PA (216) Amino acids in Metabolomics: Perspective for the Use of Regulatory effects of Fee Amino Acids in the Creation on their Basis of Infusion Solutions. Int $J$ Hematol Ther 2: 1-2.

20. Karavay A (2016) Metabolic Comfort in Oncology and Free Amino Acids: Perspectives for the Use of Their Regulatory Actions in Physiological Concentrations. Academic Web Journal of Medical Science 1: 4

Copyright: (C2017 Nefyodov L. This is an open-access article distributed under the terms of the Creative Commons Attribution License, which permits unrestricted use, distribution, and reproduction in any medium, provided the original author and source are credited. 